\title{
Unraveling the Orbital Physics in a Canonical Orbital System $\mathrm{KCuF}_{3}$
}

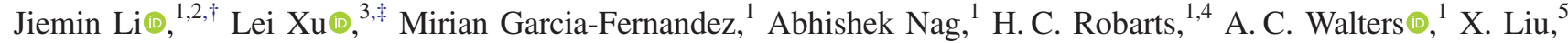 \\ Jianshi Zhou, ${ }^{6}$ Krzysztof Wohlfeld $\odot,{ }^{7}$ Jeroen van den Brink, ${ }^{3,8}$ Hong Ding, ${ }^{2}$ and Ke-Jin Zhou $\odot^{1, *}$ \\ ${ }^{1}$ Diamond Light Source, Harwell Campus, Didcot OX11 ODE, United Kingdom \\ ${ }^{2}$ Beijing National Laboratory for Condensed Matter Physics and Institute of Physics, Chinese Academy of Sciences, \\ Beijing 100190, China \\ ${ }^{3}$ Institute for Theoretical Solid State Physics, IFW Dresden, Helmholtzstrasse 20, D-01069 Dresden, Germany \\ ${ }^{4} \mathrm{H}$. H. Wills Physics Laboratory, University of Bristol, Bristol BS8 1TL, United Kingdom \\ ${ }^{5}$ School of Physical Science and Technology, ShanghaiTech University, Shanghai 201210, China \\ ${ }^{6}$ The Materials Science and Engineering Program, Mechanical Engineering, University of Texas at Austin, Austin, Texas 78712, USA \\ ${ }^{7}$ Institute of Theoretical Physics, Faculty of Physics, University of Warsaw, Pasteura 5, PL-02093 Warsaw, Poland \\ ${ }^{8}$ Institute for Theoretical Physics and Würzburg-Dresden Cluster of Excellence ct.qmat, TU Dresden, 01069 Dresden, Germany
}

(Received 8 September 2020; revised 16 December 2020; accepted 21 January 2021; published 9 March 2021)

\begin{abstract}
We explore the existence of the collective orbital excitations, orbitons, in the canonical orbital system $\mathrm{KCuF}_{3}$ using the $\mathrm{Cu} L_{3}$-edge resonant inelastic x-ray scattering. We show that the nondispersive highenergy peaks result from the $\mathrm{Cu}^{2+} d d$ orbital excitations. These high-energy modes display good agreement with the $a b$ initio quantum chemistry calculation, indicating that the $d d$ excitations are highly localized. At the same time, the low-energy excitations present clear dispersion. They match extremely well with the two-spinon continuum following the comparison with Müller ansatz calculations. The localized $d d$ excitations and the observation of the strongly dispersive magnetic excitations suggest that the orbiton dispersion is below the resolution detection limit. Our results can reconcile with the strong local Jahn-Teller effect in $\mathrm{KCuF}_{3}$, which predominantly drives orbital ordering.
\end{abstract}

DOI: 10.1103/PhysRevLett.126.106401

Introduction.-Similar to the spin or charge ordering, the electron orbital can form long-range ordering in strongly correlated materials [1]. For example, the colossal magnetoresistive manganite presents unusual transport properties that appear to be connected to its spin and orbital order coupling [2]. In vanadates, orbital order $(\mathrm{OO})$ is known to be related to the multiple temperature-induced magnetization [3]. As one of few pseudocubic perovskite systems, $\mathrm{KCuF}_{3}$ has been reported to form a long-range $\mathrm{OO}$ at a temperature of about $800 \mathrm{~K}$ and undergo a three-dimensional (3D) antiferromagnetic (AFM) ordering below $T_{N}$ of $38 \mathrm{~K}$ [4-8]. Along with manganites, $\mathrm{KCuF}_{3}$ is generally considered to be the most prototypical orbital ordered system.

The signature of the long-range spin order is the collective spin wave due to the superexchange interaction. Similarly, Kugel and Khomskii proposed the spin-orbital superexchange coupling from which a collective orbital excitation orbiton should in principle be concomitant with the presence of the $\mathrm{OO}$ in a correlated system [9]. One of

Published by the American Physical Society under the terms of the Creative Commons Attribution 4.0 International license. Further distribution of this work must maintain attribution to the author(s) and the published article's title, journal citation, and DOI. the best studied 3D OO systems is $\mathrm{LaMnO}_{3}$ in which the dispersive orbiton has been theoretically predicted [10]. Raman scattering reported the orbitons in $\mathrm{LaMnO}_{3}$, however, its existence has not been verified by other experiments, possibly due to the complex multiplet structure of the manganese ions $[11,12]$. Thus, it is instructive to look at the other prototypical orbital system, $\mathrm{KCuF}_{3}$, which is actually far simpler. Here the $\mathrm{OO}$ is generally accepted to be largely driven by the Jahn-Teller (JT) effect [13]. The search for orbitons by nonresonant inelastic $\mathrm{x}$-ray scattering did not reveal any evidence of the orbitons mode in the energy range up to $120 \mathrm{meV}$. It has been argued, though, that the orbitons may exist at a much higher energy range [14]. High-energy excitations have been studied by $\mathrm{Cu}$ $K$-edge resonant inelastic x-ray scattering (RIXS), but these studies contained no discussions of the existence of orbitons [15]. Interestingly, a large orbiton dispersion has been observed in various spin-orbital entangled cuprates and titanates by resonant inelastic soft $x$-ray scattering at the $L$ edges of transition metals owing to $\mathrm{x}$ ray's sensitivity to spin and orbital excitations [16-21].

In this Letter, we employ high-energy-resolution RIXS to explore the existence of orbitons in $\mathrm{KCuF}_{3}$ at the $\mathrm{Cu} L_{3}$ edge. $L$-edge RIXS is a well-established method for directly probing the $d d$ orbital excitations and the collective orbital and magnetic excitations in transition metal oxides 
[16-19,22-24]. It is therefore ideal to apply $L$-edge RIXS to shed light on the orbital physics in $\mathrm{KCuF}_{3}$ in both the low- and high-energy regimes. At high energy, $d d$ excitations from nondegenerated $\mathrm{Cu}^{2+} 3 d$ orbitals are resolved. They are nondispersive in the reciprocal space but demonstrate remarkable evolution in intensity. Using an $a b$ initio quantum chemistry calculation based on a single $\mathrm{CuF}_{6}$ cluster, we reproduced $d d$ excitations successfully, indicating that the local crystal-field splitting induced by a JT distortion dominates the high-energy $d d$ excitations rather than the collective orbitons. At the low-energy range, dispersive excitations are clearly resolved. Through Müller ansatz calculations, we conclude the dispersive excitations in the low-energy range are dominated by the two-spinon continuum. Our results suggest that orbitons, if they exist, may appear at a much lower energy scale than theoretically expected.

Experimental details.-Single-crystal $\mathrm{KCuF}_{3}$ compounds were prepared by the method described in Ref. [25]. A pristine sample with the surface normal (0 0 1) was selected and characterized by a lab-based Laue diffractrometer prior to the RIXS measurements. We confirm the sample has the type- $a$ orbital order structure (Fig. S1 in the Supplemental Material [26]). The RIXS experiments were conducted at the I21-RIXS beam line at Diamond Light Source, United Kingdom [38]. The sample

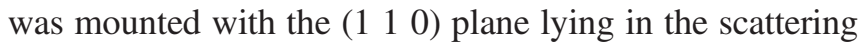
plane, as shown in Fig. 1(b). The $2 \theta$ scattering angle was fixed at $146^{\circ}$ throughout the experiment. RIXS measurements were also performed with the $\left(\begin{array}{lll}1 & 0 & 0\end{array}\right)$ plane lying in the scattering plane. The measuring temperature was kept at $16 \mathrm{~K}$ unless stated otherwise. We tuned the incident photon energy to the resonance of the $\mathrm{Cu} L_{3}$ absorption peak [see Fig. 1(a)] with either linear-horizontal $(\sigma)$ or linear-vertical $(\pi)$ polarizations for RIXS measurements. The total energy resolution is about $37 \mathrm{meV}$ FWHM. RIXS signals were collected without polarization analysis. For all RIXS spectra, the elastic (zero-energy loss) peak positions were determined by the elastic scattering spectrum from carbon tape near the sample surface and then fine adjusted by the Gaussian fitted elastic peak position. All RIXS spectra are normalized by the integrated intensities from the highenergy region $(0.5 \mathrm{eV}-2 \mathrm{eV})$. The Miller indices in this study are defined by a pseudotetragonal unit cell with $a=b \simeq 4.146 \AA$ and $c \simeq 3.92 \AA$. The momentum transfer $\boldsymbol{q}$ is defined in reciprocal lattice units (r.l.u.) as $\boldsymbol{q}=h \boldsymbol{a}^{*}+$ $k \boldsymbol{b}^{*}+l \boldsymbol{c}^{*}$ where $\boldsymbol{a}^{*}=2 \pi / a$, etc.

Results and discussion.-Figure 1(a) shows the $\mathrm{Cu}$ $L_{3}$-edge $\mathrm{x}$-ray absorption spectra (XAS) of $\mathrm{KCuF}_{3}$ excited by two linear polarizations. The main peak at $932.5 \mathrm{eV}$ corresponds to the $2 p^{5} 3 d^{10}$ final state, and the shoulder peak at about $935 \mathrm{eV}$ stems from the $2 p^{5} 3 d^{10} \underline{L}$ state $(\underline{L}$ represents a hole at ligand- $F$ site) [39]. The comparable $\mathrm{Cu}$ $L_{3}$-edge XAS intensity demonstrates the $3 \mathrm{D}$ character of the orbital ground state. A representative RIXS spectrum (a)

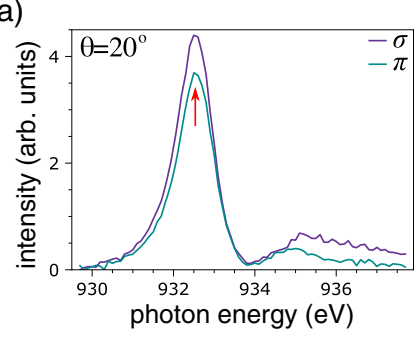

(b)

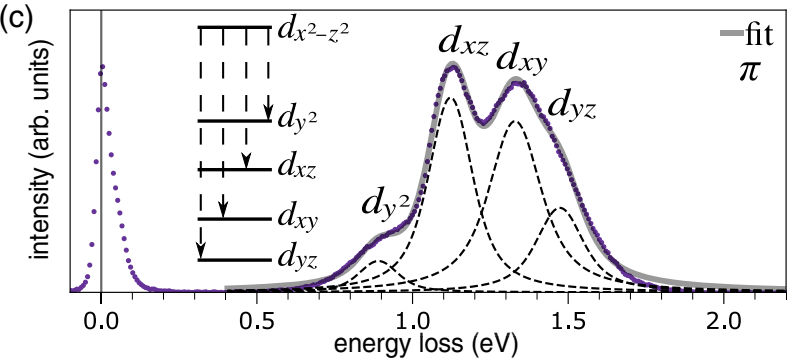

FIG. 1. Scattering geometry and overview of RIXS spectra. (a) $\mathrm{Cu} L_{3}$-XAS spectra of $\mathrm{KCuF}_{3}$ collected with the partial fluorescence yield. (b) A sketch of the experimental geometry. Light blue arrows represent the incident $\left(k_{i}\right)$ and scattered $\left(k_{f}\right) \mathrm{x}$ rays, while the double arrows (green: $\pi$, purple: $\sigma$ ) are for the polarizations of incident $\mathrm{x}$-rays. Red arrows indicate the momentum transfer and the corresponding projection parallel and perpendicular to the sample surface. Crystal axes are represented by black arrows. (c) A fitting example of the $d d$ excitations. The purple dotted line is an experimental spectrum, and the gray solid line represents the total fit. The inset depicts the energy splitting of $3 d$ orbitals.

excited by $\pi$ polarized x-rays is shown in Fig. 1(c), which comprises two regions: a high-energy $d d$ excitation that splits to four peaks, and a low-energy excitation region.

We first address the high-energy excitations. The $d d$ orbital excitations in $\mathrm{KCuF}_{3}$, though having a comparable energy scale with respect to that of two-dimensional cuprates [22], possess different energy splitting owing to the low $\mathrm{D}_{2 h}$ crystal-field symmetry. We show the $3 d$ orbitals splitting in the inset of Fig. 1(c), where the ground state holds a $d_{x^{2}-z^{2}}$ hole orbital given the definition of the $x y z$ axes with respect to the crystal orientation [26]. The rotation of local distortion along each of three axes then induces the $d_{y^{2}-z^{2}}$ hole orbital at the next site, thus introducing a 3D long-range OO [40]. As demonstrated in Fig. 1(c), orbital excitations are resolved to four peaks labeled with orbital characters as sketched in the inset of Fig. 1(c). We fitted the orbital excitations with a model comprising four Lorentzian functions convoluted by Gaussian energy resolution. Together we plot the fitted $d d$ peaks. The fitted energy positions are found to be comparable to optical and the $\mathrm{Cu} K$-edge RIXS studies (see Table I in Ref. [26]) [15,41].

To further study the high-energy orbital excitations, we performed RIXS measurements by varying the incident $\theta$ angle from $15^{\circ}$ to $140^{\circ}$. The results are shown in Fig. 2(a) 

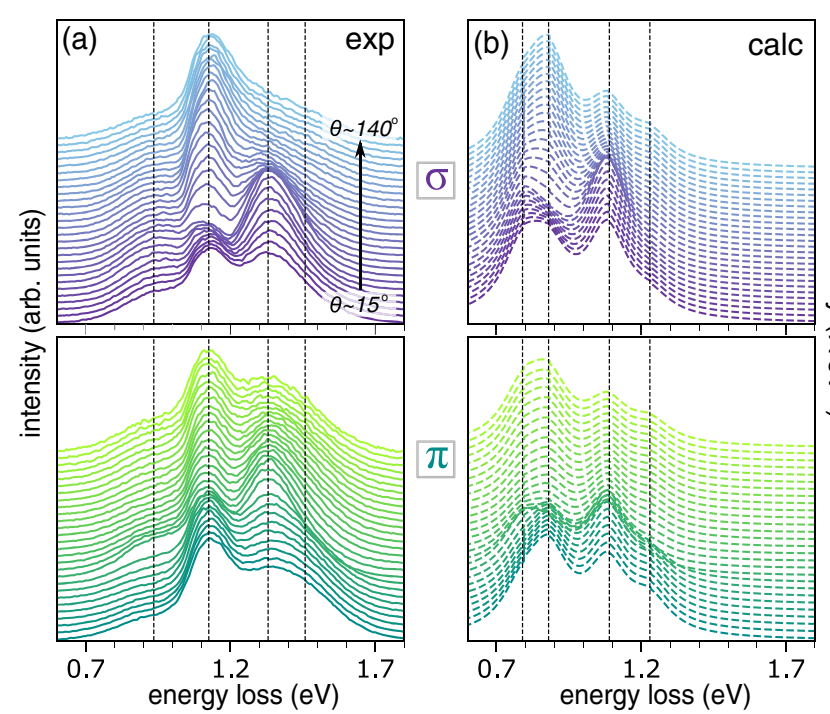

FIG. 2. Angular dependence of $d d$ excitations. (a) Experimental results $\sigma$ - (purple) and $\pi$ polarized (green) incident x-rays. The vertical dashed lines depict the averaged peak values of fitted $d d$ excitations. (b) Calculated angular-dependent spectra from the MRCI + SOC. The vertical dashed lines show the calculated values of $d d$ excitations.

with the top and the bottom figures from the $\sigma$ and $\pi$ polarizations, respectively. Assisted by the fitting analysis, we reach the conclusion that all $d d$ excitations are nondispersive but exhibit a rich intensity variation as a function of $\theta$ (Fig. S2 in the Supplemental Material [26]). Similar to other cuprate compounds, the behavior in $d d$ excitations is known to be induced by the local ligand-field splitting [22].

To understand better the $d d$ excitations, we carried out the $a b$ initio quantum chemistry calculations using the complete active space self-consistent field and multireference configuration interaction (MRCI) as implemented in the MOLPRO package [32]. An embedded cluster consisting of a single $\mathrm{CuF}_{6}$ octahedron (one $\mathrm{Cu}$ atom and six $\mathrm{F}$ atoms, with short and long bonding lengths in the $a b$ plane) was considered in the calculations, using the crystallographic data as reported in Ref. [6]. In the MRCI treatment, the F $2 p$ and $\mathrm{Cu} 2 s, 2 p, 3 s, 3 p, 3 d$ electrons within the single $\mathrm{CuF}_{6}$ unit were correlated. Details about the computed orbital excitation energies and the comparison with the experimental values are given in Ref. [26]. To account for the orbital ordering effect, the calculations were performed for both $d_{x^{2}-z^{2}}$ and $d_{y^{2}-z^{2}}$ hole orbitals. The latter was achieved by rotating the $\mathrm{CuF}_{6}$ octahedron around the $c / z$ axis by $90^{\circ}$.

Figure 2(b) shows the averaged theoretical spectra as a function of $\theta$ based on the MRCI plus spin-orbit coupling (SOC) approach. The results for both polarizations agree well with the experimental spectra. To make a more detailed comparison, we fitted all spectra and extracted the area of each orbital excitation. The intensities of each orbital excitation from the experimental and theoretical

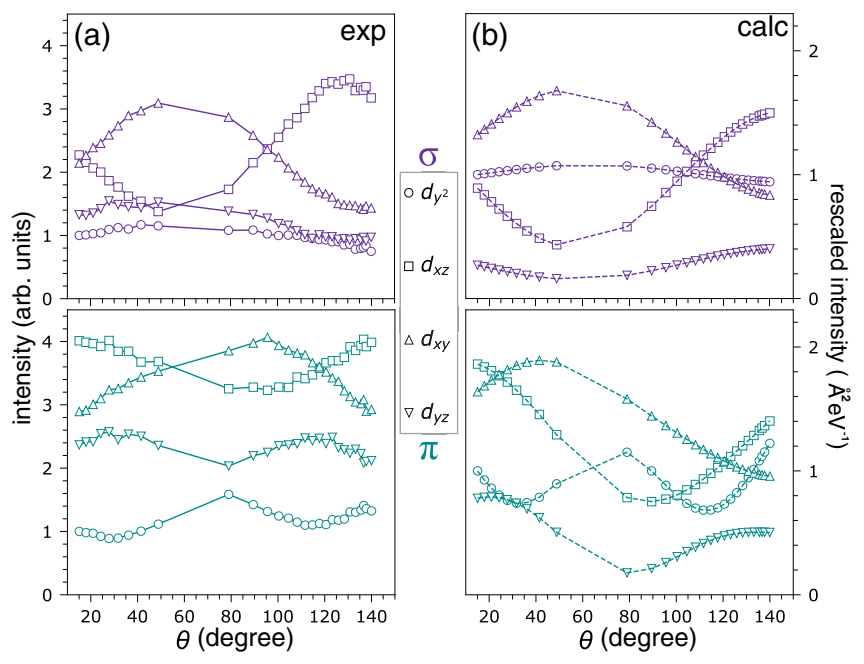

FIG. 3. Relative intensity variations as a function of $\theta$ angle for different $d d$ excitations. (a) is the experimental results; (b) is the calculated results. The first data point of the $d_{y^{2}}$ orbital is normalized to a fixed intensity.

results are displayed in Fig. 3(a), (b), respectively. The comparison shows fairly good agreement in terms of the trend of the angular-dependent intensity except for the $d_{y z}$ $\left(d_{x y}\right)$ orbital in the $\sigma(\pi)$ polarization. The difference in the $d_{y z}$ orbital may be due to the uncertainty of determining the intensity because of the dominant $d_{x y}$ orbital. For the $d_{x y}$ orbital in the $\pi$ polarization, a repeated RIXS measurement shows consistent angular dependence. To cross check the theoretical results, we performed independent calculations using the single-ion model [22]. Interestingly, the results are consistent with the quantum chemistry calculations except for the $d_{x y}$ orbital in the $\pi$ polarization, which shows a maximal intensity around $\theta$ of $70^{\circ}$ (Fig. S6 in the Supplemental Material [26]). Therefore, the discrepancy likely stems from the simplification of the single cluster model. We note that the averaged results are similar to those derived from the single $d_{x^{2}-z^{2}}$ or $d_{y^{2}-z^{2}}$ hole orbital state (Fig. S3 in the Supplemental Material [26]). Remarkably, the experimental results acquired under the $\left(\begin{array}{lll}1 & 0 & 0\end{array}\right)$ geometry could only be supported by the averaged theoretical spectra than the spectra from either $d_{x^{2}-z^{2}}$ or $d_{y^{2}-z^{2}}$ single state (Figs. S4 and S5 in the Supplemental Material [26]). We therefore conclude that the high-energy $d d$ excitations are consistent with the local JT effect and the orbital ordering of the system. Altogether, the strongly localized nondispersive $d d$ excitations do not seem to support the existence of collective orbitons at such high-energy ranges [14].

We now turn to the discussion of the low-energy excitations to further explore the potential existence of the collective orbiton. In Figs. 4(a) and 4(b), we display the maps of the angular-dependent low-energy excitations probed by the linear $\sigma$ and $\pi$ polarizations, respectively. Both maps show excitations from zero loss energy up to 

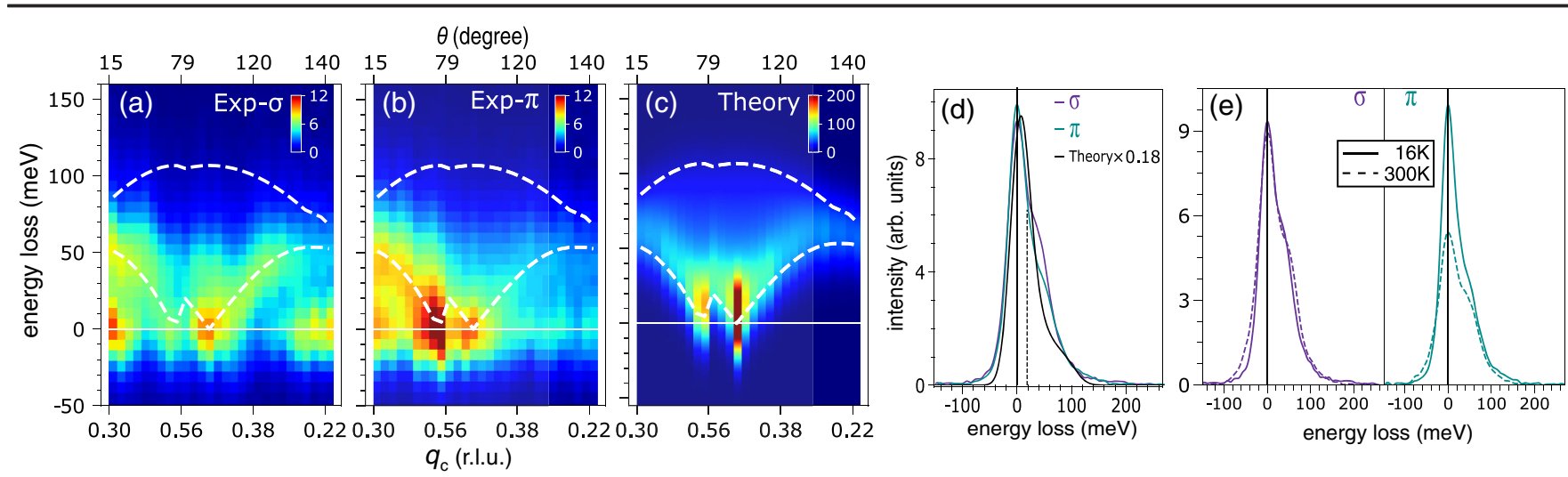

FIG. 4. Low-energy excitations of $\mathrm{KCuF}_{3}$ revealed by the $\mathrm{Cu} L_{3}$ edge RIXS. (a) and (b) are color maps of the low-energy RIXS spectra from the $\sigma$ and $\pi$ polarizations, respectively. (c) is the Müller ansatz calculated results. The white dashed lines are the lower and upper boundaries of the two-spinon continuum. The thin white line is the zero energy reference. (d) The comparison of RIXS spectra at $q_{c}=0.5$ r.l.u. The dashed line marks the longitudinal magnetic mode observed in INS [42]. (e) Temperature-dependent RIXS spectra at $q_{c}=0.5$ r.l.u. for different polarizations.

about $100 \mathrm{meV}$, where a mode emanates from $\theta \simeq 100^{\circ}$ and disperses to higher energy by approaching to either side of the $\theta$ range. Specifically, near a $\theta$ of $80^{\circ}$, two maximal intensity points are present at the zero loss energy positions. In $\mathrm{KCuF}_{3}$, the well-known dispersive modes are the twospinon continuum reported by the inelastic neutron scattering (INS) because of the quasi-one-dimensional magnetic properties [4,6,8]. Given the sensitivity of RIXS to magnetic excitations, we corroborate that the observed dispersive modes in RIXS are dominated by the two-spinon continuum.

To verify the assignment, we analyze more quantitatively the spin dynamics in $\mathrm{KCuF}_{3}$ using the Müller ansatz [43-46]. As established in Ref. [47], the lower and upper boundaries of the two-spinon continuum can be expressed by the following sinusoidal functions:

$$
E_{l}\left(q_{c}\right)=\frac{\pi}{2} J_{c}\left|\sin q_{c}\right| \quad \text { and } \quad E_{u}\left(q_{c}\right)=\pi J_{c}\left|\sin \frac{q_{c}}{2}\right|,
$$

where $q_{c}$ is the projected wave vector along the $c$ axis in the unit of r.l.u., and $J_{c}$ is the AFM superexchange interaction. As a good approximation, the magnetic dynamic structure factor at $T=0 \mathrm{~K}$ can be expressed as [46]

$$
S(E, q)=\frac{289.6}{\pi} \frac{H\left[E-E_{l}(q)\right] \times H\left[E_{u}(q)-E\right]}{\sqrt{E^{2}-E_{l}(q)^{2}}} .
$$

Here, $H[x]$ is the Heaviside step function. We evaluated this expression using $J_{c}=34 \mathrm{meV}$, which is based on the INS results [47]. Before we plot the calculated spectra, we note that the photon momentum transfer along the sample $c$ axis passes through the AFM wave vector $q_{c}=0.5$ r.l.u. twice under the fixed RIXS scattering configuration [26]. Correspondingly, we show theoretical results as a function of $q_{c}$ in Fig. 4(c) [26]. In Figs. 4(a) and 4(b), we plot the momentum transfer $q_{c}$ at the bottom axes and superimpose the two-spinon continuum lower and upper boundaries (white dashed lines) on top of the experimental results. Remarkably, the center of mass of the RIXS low-energy excitations matches the lower limit of two-spinon dispersions extremely well. In particular, two maximal intensity spots near the zero loss energy position agree precisely with the theoretical results.

Comparing the RIXS results to the INS data [47], some extra spectral weight seems to exist specifically near $q_{c}=0.5$ r.l.u. We plot the corresponding line spectra in Fig. 4(d) together with the theoretical result. The peak position of the mode appears at $\sim 40$ (47) $\mathrm{meV}$ for the $\sigma(\pi)$ polarizations, which is absent in the theory. The longitudinal magnetic mode, i.e., the signature of the 3D magnetic ordering, should in principle exist in RIXS spectra as the experiments were conducted below $T_{N}$ of $38 \mathrm{~K}$ [42]. However, its center energy of $\sim 18.5 \mathrm{meV}$ is below the RIXS energy resolution. On the other hand, the mode seems to be comparable to the optical phonon observed by Raman [48] and IXS [14]. To further explore the origin of the mode, we performed temperature-dependent measurements at $q_{c}=0.5$ r.l.u. The data are shown in Fig. 4(e). The persistence of the peak up to room temperature in both polarizations confirms the phonon-like origin of this low-energy mode at $q_{c}=0.5$ r.l.u.

We now address the relevance of the observed lowenergy excitations to the expected dispersive orbiton in $\mathrm{KCuF}_{3}$. It is understood that the spin-orbital exchange of purely electronic origin should not be considered the mere mechanism that is responsible for the orbiton dispersion. As the $\mathrm{OO}$ is mostly driven by the JT mechanism whose Hamiltonian has an identical form to the orbital part of the superexchange interaction [9,49-51], the orbiton dispersion should mainly result from the JT effect. This is consistent with what was suggested in Ref. [48], which is that the orbital-only interaction, which comprises the JT and the on-ligand interaction, is about $600 \mathrm{~K}(\sim 52 \mathrm{meV})$. 
By taking into account also the effective spin-orbital exchange of electronic origin $(\sim 3 \mathrm{meV})$, we estimate approximately the total orbital superexchange $\left(J_{\mathrm{OO}}\right)$ to be about $55 \mathrm{meV}$ along the $c$ direction, which could result in an orbiton bandwidth of $\sim 110 \mathrm{meV}$. Such a large bandwidth would yield an obvious dispersive orbiton given the experimental energy resolution of $37 \mathrm{meV}$.

Interestingly, we found an excellent agreement between the low-energy RIXS spectra and the Müller ansatz calculations, which indicates that the orbiton dispersion must be well below the dispersive two-spinon continuum. It is thus puzzling to understand why the orbital superexchange $\left(J_{\mathrm{OO}} \sim 55 \mathrm{meV}\right)$ induced JT coupling does not lead to a sizeable and observable orbiton dispersion. While we leave a detailed answer to this question for a future work, we here suggest the following explanation. Apart from the cooperative, global JT effect and ordering, the local JT effect, i.e., the JT coupling between the orbital degrees of freedom and the local lattice vibrations, is of prime importance [51,52]. As discussed in Ref. [51], the latter effect, which should be present in any JT active system, can lead to a strong dressing of the orbitons with local vibrational modes and may cause a complete smearing out of the orbiton dispersion, cf. Fig. 5(b) of [51]. Since the JT coupling is inherently strong in $\mathrm{KCuF}_{3}$, we believe this scenario explains the effective disappearance of the orbiton dispersion in this system. Our work is significant in recognizing the importance of the dressing effect of the local JT distortion on the collective orbiton in $\mathrm{KCuF}_{3}$ and many other orbital-ordered systems with strong JT effects.

Conclusion.-In summary, we performed high-resolution RIXS experiments on the orbitally ordered $\mathrm{KCuF}_{3}$. The high-energy excitations are found to stem from localized $d d$ orbital excitations, consistent with the ab initio calculation based on a single cluster. The low-energy dispersive excitations are dominated by the two-spinon continuum via the comparison to Müller ansatz calculations. This indicates that the relevant energy bandwidth of the orbitons may be much lower than the energy resolution of RIXS experiments. We suggest that the main reason for the lack of the onset of an orbiton with a dispersion above the resolution threshold lies in the possibly strong local JT effect, which may lead to the dressing of the orbiton with the local vibrational modes and thus to the suppression of the orbiton dispersion.

We thank E. Pavarini for fruitful discussions. We also thank Nikolay Bogdanov for the assistance on the theoretical models. J. L. acknowledges Diamond Light Source (United Kingdom) and the Institute of Physics in Chinese Academy of Sciences (China) for providing funding Grant No. 112111KYSB20170059 for the joint Doctoral Training under the contract STU0171 and also the financial support from China Scholarship Council. L. X. thanks U. Nitzsche for technical assistance. X. L. was supported by National Natural Science Foundation of China under Grant
No. 11934017. J.S.Z. acknowledges the support by NSF Grant No. DMR-1905598 in the United States. K. W. acknowledges support by Narodowe Centrum Nauki (NCN) Projects No. 2016/22/E/ST3/00560 and No. 2016/23/B/ST3/00839. J. v. d. B. acknowledges financial support through the Deutsche Forschungsgemeinschaft (DFG, German Research Foundation), SFB 1143 project A5, and through the Wurzburg-Dresden Cluster of Excellence on Complexity and Topology in Quantum Matter-ct.qmat (EXC 2147, project-id 39085490). H. D. acknowledges support by the National Natural Science Foundation of China (No. 11888101) and the Ministry of Science and Technology of China (2016YFA0401000). All data were taken at the I21 RIXS beam line of Diamond Light Source (United Kingdom) using the RIXS spectrometer designed, built, and owned by Diamond Light Source. We acknowledge Diamond Light Source for providing the science commissioning time on Beamline I21. We acknowledge Thomas Rice for the technical support throughout the beam time. We would also like to thank the Materials Characterization Laboratory team for help on the Laue instrument in the Materials Characterization Laboratory at the ISIS Neutron and Muon Source.

J. L. and L. X. contributed equally to this work.

* Corresponding author.

kejin.zhou@diamond.ac.uk

Present address: National Synchrotron Light Source II, Brookhaven National Laboratory, Upton, New York 11973, USA.

*Present Address: Theoretical Division, Los Alamos National Laboratory, Los Alamos, New Mexico 87544, USA.

[1] Y. Tokura and N. Nagaosa, Science 288, 462 (2000).

[2] C. D. Ling, J. E. Millburn, J. F. Mitchell, D. N. Argyriou, J. Linton, and H. N. Bordallo, Phys. Rev. B 62, 15096 (2000).

[3] Y. Ren, T. T. M. Palstra, D. I. Khomskii, E. Pellegrin, A. A. Nugroho, A. A. Menovsky, and G. A. Sawatzky, Nature (London) 396, 441 (1998).

[4] M. T. Hutchings, E. J. Samuelsen, G. Shirane, and K. Hirakawa, Phys. Rev. 188, 919 (1969).

[5] N. Tsukuda and A. Okazaki, J. Phys. Soc. Jpn. 33, 1088 (1972).

[6] R. Caciuffo, L. Paolasini, A. Sollier, P. Ghigna, E. Pavarini, J. van den Brink, and M. Altarelli, Phys. Rev. B 65, 174425 (2002).

[7] L. Paolasini, R. Caciuffo, A. Sollier, P. Ghigna, and M. Altarelli, Phys. Rev. Lett. 88, 106403 (2002).

[8] S. K. Satija, J. D. Axe, G. Shirane, H. Yoshizawa, and K. Hirakawa, Phys. Rev. B 21, 2001 (1980).

[9] K. I. Kugel and D. I. Khomskii, Sov. Phys. Usp. 25, 231 (1982).

[10] J. van den Brink, P. Horsch, F. Mack, and A. M. Olés, Phys. Rev. B 59, 6795 (1999).

[11] E. Saitoh, S. Okamoto, K. T. Takahashi, K. Tobe, K. Yamamoto, T. Kimura, S. Ishihara, S. Maekawa, and Y. Tokura, Nature (London) 410, 180 (2001). 
[12] M. Grüninger, R. Rückamp, M. Windt, P. Reutler, C. Zobel, T. Lorenz, A. Freimuth, and A. Revcolevschi, Nature (London) 418, 39 (2002).

[13] E. Pavarini, E. Koch, and A. I. Lichtenstein, Phys. Rev. Lett. 101, 266405 (2008).

[14] Y. Tanaka, A. Q. R. Baron, Y.-J. Kim, K. J. Thomas, J. P. Hill, Z. Honda, F. Iga, S. Tsutsui, D. Ishikawa, and C. S. Nelson, New J. Phys. 6, 161 (2004).

[15] K. Ishii, S. Ishihara, Y. Murakami, K. Ikeuchi, K. Kuzushita, T. Inami, K. Ohwada, M. Yoshida, I. Jarrige, N. Tatami, S. Niioka, D. Bizen, Y. Ando, J. Mizuki, S. Maekawa, and Y. Endoh, Phys. Rev. B 83, 241101(R) (2011).

[16] J. Schlappa, K. Wohlfeld, K. J. Zhou, M. Mourigal, M. W. Haverkort, V. N. Strocov, L. Hozoi, C. Monney, S. Nishimoto, S. Singh, A. Revcolevschi, J. S. Caux, L. Patthey, H. M. Rønnow, J. van den Brink, and T. Schmitt, Nature (London) 485, 82 (2012).

[17] V. Bisogni, K. Wohlfeld, S. Nishimoto, C. Monney, J. Trinckauf, K. Zhou, R. Kraus, K. Koepernik, C. Sekar, V. Strocov, B. Büchner, T. Schmitt, J. van den Brink, and J. Geck, Phys. Rev. Lett. 114, 096402 (2015).

[18] R. Fumagalli, J. Heverhagen, D. Betto, R. Arpaia, M. Rossi, D. Di Castro, N. B. Brookes, M. Moretti Sala, M. Daghofer, L. Braicovich, K. Wohlfeld, and G. Ghiringhelli, Phys. Rev. B 101, 205117 (2020).

[19] C. Ulrich, G. Ghiringhelli, A. Piazzalunga, L. Braicovich, N. B. Brookes, H. Roth, T. Lorenz, and B. Keimer, Phys. Rev. B 77, 113102 (2008).

[20] K. Wohlfeld, M. Daghofer, S. Nishimoto, G. Khaliullin, and J. van den Brink, Phys. Rev. Lett. 107, 147201 (2011).

[21] K. Wohlfeld, S. Nishimoto, M. W. Haverkort, and J. van den Brink, Phys. Rev. B 88, 195138 (2013).

[22] M. Moretti Sala, V. Bisogni, C. Aruta, G. Balestrino, H. Berger, N. B. Brookes, G. M. de Luca, D. Di Castro, M. Grioni, M. Guarise, P. G. Medaglia, F. Miletto Granozio, M. Minola, P. Perna, M. Radovic, M. Salluzzo, T. Schmitt, K. J. Zhou, L. Braicovich, and G. Ghiringhelli, New J. Phys. 13, 043026 (2011).

[23] L. Braicovich, J. van den Brink, V. Bisogni, M. M. Sala, L. J. P. Ament, N. B. Brookes, G. M. De Luca, M. Salluzzo, T. Schmitt, V. N. Strocov, and G. Ghiringhelli, Phys. Rev. Lett. 104, 077002 (2010).

[24] L. J. P. Ament, M. van Veenendaal, T. P. Devereaux, J. P. Hill, and J. van den Brink, Rev. Mod. Phys. 83, 705 (2011).

[25] L. G. Marshall, J. Zhou, J. Zhang, J. Han, S. C. Vogel, X. Yu, Y. Zhao, M. T. Fernández-Díaz, J. Cheng, and J. B. Goodenough, Phys. Rev. B 87, 014109 (2013).

[26] See Supplemental Material, which includes Refs. [27-37], at http://link.aps.org/supplemental/10.1103/PhysRevLett .126 .106401 for more details about the sample information, the experimental and theoretical $d d$ orbital excitations, and the simulation of low-energy spin excitations.
[27] R. H. Buttner, E. N. Maslen, and N. Spadaccini, Acta Crystallogr. B46, 131 (1990).

[28] M. Klintenberg, S. Derenzo, and M. J. Weber, J. Comput. Phys. Commun. 131, 120 (2000).

[29] N. A. Bogdanov, V. Bisogni, R. Kraus, C. Monney, K. Zhou, T. Schmitt, J. Geck, A. O. Mitrushchenkov, H. Stoll, J. van den Brink, and L. Hozoi, J. Phys. Condens. Matter 29, 035502 (2017).

[30] N. B. Balabanov and K. A. Peterson, J. Chem. Phys. 123, 064107 (2005)

[31] T. H. Dunning Jr., J. Chem. Phys. 90, 1007 (1989).

[32] T. Helgaker, P. Jørgensen, and J. Olsen, Molecular Electronic-Structure Theory (Wiley, Chichester, 2000).

[33] J. Pipek and P. G. Mezey, J. Chem. Phys. 90, 4916 (1989).

[34] P. J. Knowles and H.-J. Werner, Theor. Chim. Acta 84, 95 (1992).

[35] H.-J. Werner and P. J. Knowles, J. Chem. Phys. 89, 5803 (1988).

[36] A. Mitrushchenkov and H.-J. Werner, Mol. Phys. 105, 1239 (2007).

[37] M. van Veenendaal, Theory of Inelastic Scattering and Absorption of X-Rays (Cambridge University Press, Cambridge, England, 2015), pp. 112-118.

[38] https://www.diamond.ac.uk/Instruments/MagneticMaterials/I21.html.

[39] C. De Nadaï, A. Demourgues, J. Grannec, and F. M. F. de Groot, Phys. Rev. B 63, 125123 (2001).

[40] M. Hidaka, T. Eguchi, and I. Yamada, J. Phys. Soc. Jpn. 67, 2488 (1998).

[41] J. Deisenhofer, I. Leonov, M. V. Eremin, Ch. Kant, P. Ghigna, F. Mayr, V. V. Iglamov, V. I. Anisimov, and D. van der Marel, Phys. Rev. Lett. 101, 157406 (2008).

[42] B. Lake, D. A. Tennant, and S. E. Nagler, Phys. Rev. B 71, 134412 (2005).

[43] J. des Cloizeaux and J. J. Pearson, Phys. Rev. 128, 2131 (1962).

[44] L. D. Faddeev and L. A. Takhtajan, Phys. Lett. 85A, 375 (1981).

[45] F. D. M. Haldane and M. R. Zirnbauer, Phys. Rev. Lett. 71, 4055 (1993).

[46] G. Müller, H. Thomas, H. Beck, and J. C. Bonner, Phys. Rev. B 24, 1429 (1981).

[47] B. Lake, D. A. Tennant, C. D. Frost, and S. E. Nagler, Nat. Mater. 4, 329 (2005).

[48] J. C. T. Lee et al., Nat. Phys. 8, 63 (2012).

[49] S. Okamoto, S. Ishihara, and S. Maekawa, Phys. Rev. B 66, 014435 (2002).

[50] J. van den Brink, New J. Phys. 6, 201 (2004).

[51] J. Nasu and S. Ishihara, Phys. Rev. B 88, 205110 (2013).

[52] J. van den Brink, Phys. Rev. Lett. 87, 217202 (2001). 